\title{
Experiencia de innovación educativa de aula invertida en asignatura de Máster en Ingeniería Energética
}

\section{Flipped Classroom educational innovation experience in a Master in Energy Engineering subject}

\author{
A. Meana-Fernández¹, B. Peris-Pérez², J.C. Ríos-Fernández³, J.M. González- \\ Caballín ${ }^{4}$ \& A.J. Gutiérrez-Trashorras ${ }^{5}$
}

Fecha de recepción: 27/02/2020; Fecha de revisión: 25/03/2020; Fecha de aceptación: 03/09/2020

Cómo citar este artículo:

Meana-Fernández, A., Peris-Pérez, J.C., Ríos-Fernández, J.C., González-Caballín, J.M. \& GutiérrezTrashorras, A.J. (2020). Experiencia de innovación educativa de aula invertida en asignatura de Máster en ingeniería Energética. Revista de Innovación y Buenas Prácticas Docentes, 9(2), 61-70.

Autor de Correspondencia: andresmf@uniovi.es

\begin{abstract}
Resumen:
En este trabajo se describe la experiencia de innovación llevada a cabo en la asignatura de Exergía, Termoeconomía y Cogeneración del Máster Universitario en Ingeniería Energética de la Universidad de Oviedo durante el curso 2019/2020. Los resultados de aprendizaje mostraban que parte del potencial del estudiantado se encontraba desaprovechado, por lo que se detectó la necesidad de conseguir un mejor aprovechamiento de las clases presenciales. Con este objetivo, se desarrolló un Proyecto de Innovación Docente basado en una metodología de aula invertida y de aprendizaje colaborativo, en el que los alumnos preparaban una serie de contenidos antes de acudir al aula para luego trabajar de forma colaborativa en la misma. Con dicho Proyecto, se ha conseguido mejorar sustancialmente los resultados de aprendizaje, así como lograr un mayor grado de satisfacción del estudiantado respecto a la asignatura y la metodología docente empleada. Las interacciones en el aula han mejorado notablemente y la confianza de los estudiantes en sí mismos y su conocimiento se ha reforzado. Finalmente, se recoge una autoevaluación de la mejora implementada, así como posibles propuestas para el futuro. La principal es la continuación de la mejora en el curso siguiente para poder evaluar su consolidación.
\end{abstract}

Palabras clave: aprendizaje colaborativo, aula invertida, innovación pedagógica, mejora de la docencia

\footnotetext{
1 Universidad de Oviedo (España), andresmf@uniovi.es; CÓDIGO ORCID: 0000-0002-1865-3814

2 Universidad de Sevilla (España), bperis@us.es; CÓDIGO ORCID: 0000-0001-9993-6068

${ }^{3}$ Universidad de Oviedo (España), riosjuan@uniovi.es; CÓDIGO ORCID: 0000-0002-2984-9206

${ }^{4}$ Universidad de Oviedo (España), gonzalezsjuan@uniovi.es; CÓDIGO ORCID: 0000-0002-8442-9538

5 Universidad de Oviedo (España), gutierrezantonio@uniovi.es; CÓDIGO ORCID: 0000-0002-9430-7251
} 


\section{Abstract:}

This work describes the innovation experience performed in the subject Exergy, Thermoeconomy and Cogeneration of the Master in Energy Engineering of the University of Oviedo during the year 2019/2020. After reviewing the learning results of the students and realizing that part of their potential was being lost, the necessity of improving the time management in the classroom was identified. With this aim, a Teaching Innovation Project based on a flipped classroom and collaborative learning methodology was developed, in which the students prepared a series of contents at home, before coming to class, to work collaboratively afterwards in the classroom. With this Project, the learning results have been substantially improved. Additionally, the students feel more satisfied about the subject and the teaching methodology employed. The classroom interactions have noticeably improved and the self-confidence of students on themselves and their knowledge has been reinforced. Finally, a self-assessment of the implemented improvement has been performed and future proposals have been outlined. The main proposal is to continue the implemented improvement next term to evaluate its consolidation.

Key Words: collaborative learning, flipped classroom, teaching improvement, teaching innovation 


\section{INTRODUCCIÓN}

La creación del Espacio Europeo de Educación Superior (EEES) conlleva un cambio de paradigma en el que el estudiante pasa a ser el centro del proceso educativo como su principal actor. Dicho paradigma se concreta en el abandono de las lecciones magistrales como único medio para la transmisión del conocimiento y la necesidad de evaluar permanentemente la metodología docente empleada para ajustarla a las necesidades particulares del estudiantado. De esta manera, el proceso tradicional de enseñanza se ha transformado en el binomio enseñanza-aprendizaje, haciendo hincapié en la dimensión activa del estudiantado en dicho proceso. Por este motivo, los métodos a utilizar deben actualizarse periódicamente para poder dar respuesta a los cambios en el contexto universitario y contribuir a la mejora de la calidad docente (Fernández Díaz, 2005). Además, el marco legislativo establecido por los Reales Decretos 1393/2007 de 29 de octubre, por el que se establece la ordenación de las enseñanzas universitarias oficiales; 99/2011, de 28 de enero, por el que se regulan las enseñanzas oficiales de doctorado; y 1027/2011, de 15 de julio, por el que se establece el Marco Español de Cualificaciones para la Educación Superior proporcionan una gran autonomía al profesorado a la hora de decidir cuál es la metodología que quieren aplicar en sus clases. Se puede partir de la premisa de que el aprendizaje sucede cuando los profesores ponen su energía en lo que transmiten y configuran experiencias de aprendizaje únicas y significativas (Acaso, Manzanera y Piscitelli).

Una de las metodologías que se adaptan particularmente bien a este nuevo contexto de aprendizaje es la conocida como aula invertida o "flipped classroom" en inglés (Bergmann y Sams, 2012). Esta metodología se basa en la redistribución de los tiempos en el aula y el hogar de forma que, antes de cada sesión, el estudiantado prepara una serie de contenidos en su casa referentes a la misma. Así, las sesiones en el aula ya no se emplean para la transmisión de conocimientos del docente al estudiantado de forma magistral, sino que se usa para trabajar sobre los contenidos que los estudiantes han preparado en casa antes de la misma. Dichas sesiones suelen comenzar con la resolución de dudas por parte del docente, para luego pasar a la realización de tareas y actividades por parte del estudiantado. De esta forma, el docente queda libre para poder moverse por el aula y prestar su ayuda a los estudiantes que lo necesiten. Esta metodología permite centrar el proceso de aprendizaje en cada estudiante particular (Goodwin y Miller, 2013), convirtiendo a todo el estudiantado en protagonista de su propio proceso de aprendizaje. Si bien en el campo de la innovación educativa, los mayores avances se vienen produciendo en las enseñanzas medias (Tourón y Santiago, 2015), (Romero y Prat, 2018), este modelo ya se ha aplicado en el contexto universitario con un grado de éxito satisfactorio (Pierce y Fox, 2012), (Iborra Urios et al., 2017).

El contexto generado en el aula gracias a la metodología de aula invertida facilita la introducción de técnicas de trabajo más difíciles de poner en práctica con la metodología de enseñanza tradicional. Una metodología que se puede integrar de forma beneficiosa con el aula invertida es la del aprendizaje colaborativo (Cohen y Lotan, 2014). El aprendizaje colaborativo es una metodología de aprendizaje activo basada en la interacción entre iguales, en el que el docente actúa como mediador del proceso de aprendizaje. Los estudiantes asumen una cierta responsabilidad de su propio proceso de aprendizaje (Vanwalleghem et al., 2018) y colaboran entre sí para realizar una serie de tareas, de modo que el aprendizaje obtenido es superior al que habrían obtenido en caso de trabajar de forma individual. Así, los estudiantes mejoran no solo su 
conocimiento de las materias, sino sus habilidades sociales, su capacidad de juicio crítico y se aumenta su motivación y participación, reduciendo la ansiedad y frustración.

Finalmente, el trabajo por problemas es otra de las estrategias que se pueden aplicar dentro del aula invertida. Trabajar por problemas supone un reto para el estudiantado, ya que se hace necesario que el estudiante busque entre sus propios conocimientos y experiencias para luego seleccionar aquellos que puedan ser útiles para resolver el problema. De esta forma se desarrollan sus competencias y habilidades de forma paralela a la adquisición y consolidación de conocimientos (Barrel, 2006). Este enfoque tiene bastante repercusión en el marco de las enseñanzas superiores dado que permite trabajar las competencias que los estudiantes necesitan para su posterior incorporación a la vida laboral (Maroto Molina et al., 2017). La resolución de problemas requiere tiempo y la forma de resolverlos puede ser variada, pero consigue que los estudiantes que se implican en ella lo hagan emocionalmente y da lugar a la construcción de nuevos conocimientos o a la modificación de los conocimientos existentes.

En este trabajo se describe la experiencia de innovación llevada a cabo en la asignatura de Exergía, Termoeconomía y Cogeneración del Máster Universitario en Ingeniería Energética de la Universidad de Oviedo durante el curso 2019/2020. Tras explicar la motivación de dicha experiencia, se describe el proceso de desarrollo e implantación de la misma, así como los principales resultados obtenidos. Finalmente, se realiza una autoevaluación de la mejora implementada y se presentan las principales conclusiones obtenidas.

\section{DESARROLLO DE LA EXPERIENCIA DE INNOVACIÓN}

En este apartado se describe la motivación que ha propiciado la experiencia de innovación desarrollada, así como la implantación de la misma.

\subsection{Motivación de la experiencia de innovación desarrollada}

La asignatura de Exergía, Termoeconomía y Cogeneración (ETC) es una asignatura optativa de 3 créditos ECTS, perteneciente al segundo semestre del Máster Universitario en Ingeniería Energética de la Universidad de Oviedo. En la guía docente aparece descrita como una asignatura "que aborda los aspectos teóricos fundamentales que se aplican a los procesos energéticos tradicionales, justificando las mejoras introducidas y también aquellas que podrían desarrollarse en su contexto". En este sentido, la asignatura pretende que el estudiantado sea capaz de cuantificar el rendimiento, impacto ambiental y económico asociados a diferentes procesos energéticos, en función de los recursos y de la tecnología disponibles para, de este modo, sentar las bases de la energía útil y el potencial de ahorro.

La asignatura comprende 75 horas de trabajo personal del estudiante, 22,5 de las cuales son de trabajo presencial (12 de clases expositivas, 9 de seminarios de aula y 1,5 de tutorías grupales) y 52,5 son de trabajo no presencial (27,5 para el trabajo en grupo y 25 para el trabajo individual). Para la calificación final de la asignatura se tiene en cuenta la asistencia a las clases expositivas y seminarios, en las que el estudiante realiza trabajos, realiza pruebas escritas y entrega resultados, con un peso del $40 \%$. El $60 \%$ restante se obtiene de la realización de trabajos propuestos por los docentes.

Normalmente, el $60 \%$ de los estudiantes del Máster eligen cursar esta asignatura (entre 7 y 12 estudiantes al año). El porcentaje de aprobados es muy alto, por encima del $85 \%$. Los estudiantes muestran interés por la asignatura y tienen una percepción de dificultad baja de la misma, mientras que el profesorado percibe una motivación más alta por parte del estudiantado en comparación con otras asignaturas, probablemente debido a su carácter práctico. La elevada motivación del estudiantado permite implantar estrategias innovadoras en el aula, aunque se debe tener en cuenta que la impartición de la asignatura se realiza de forma muy concentrada, a lo largo de dos semanas. 
La asignatura venía impartiéndose con una metodología tradicional, basada en clases magistrales, hasta el curso 2017/18. En el curso 2018/19, los autores de este trabajo pasaron a ser responsables de su docencia, aunque durante ese curso se continuó utilizando la misma metodología de enseñanza.

Tras observar que parte del potencial del estudiantado se encontraba desaprovechado a la luz de los resultados de aprendizaje observados, se detectó la necesidad de conseguir un mejor aprovechamiento de las clases presenciales a través de la mejora de la gestión del tiempo de aprendizaje. Con este objetivo, se desarrolló el Proyecto de Innovación "Aplicación del Método "Flipped Classroom" a asignatura del Máster Universitario en Ingeniería Energética" (PINN-19-A-101) en el marco de la convocatoria de Proyectos de Innovación Docente de la Universidad de Oviedo de 2019. El objetivo de dicho proyecto fue la introducción de una metodología docente innovadora en la asignatura basada en el aula invertida o "flipped classroom", con el fin de maximizar el grado de aprovechamiento de las clases presenciales y fomentar la capacidad de autoaprendizaje del estudiantado.

\subsection{Desarrollo e implantación en el aula de la experiencia de innovación}

El esquema de las etapas de desarrollo e implantación en el aula de la experiencia de innovación propuesta se muestra en la Figura 1. Tras la búsqueda de bibliografía y la puesta en común de ideas para implantar la experiencia, la primera fase del proyecto consistió en la elaboración de nuevo material docente a lo largo del primer cuatrimestre del curso 2019/20, previamente a la impartición de la asignatura, con el objetivo de propiciar la autonomía del estudiantado y facilitar la comprensión de los conceptos más complejos tratados en la asignatura. Se generaron nuevos contenidos teóricos y prácticos (cuestiones y problemas) que se pusieron a disposición del estudiantado a través del Campus Virtual antes de las sesiones de aula. En esta primera fase, también se generaron las encuestas de evaluación acerca de la asignatura para que el estudiantado las cumplimentara al final su impartición.

La implantación de la experiencia en el aula se realizó durante el segundo cuatrimestre del curso 2019/20. Se comenzó realizando una sesión explicativa con el estudiantado en la que, junto a la presentación de la asignatura, se introdujo la metodología "flipped classroom" y se informó a los estudiantes acerca del modo de proceder durante el transcurso de las diferentes sesiones de la asignatura. Previamente a las sesiones en el aula, se pedía a los estudiantes que trabajaran una serie de contenidos en casa. Posteriormente, las diferentes sesiones que se fueron sucediendo consistieron en una breve exposición por parte del profesor y la aclaración de dudas surgidas de la preparación de contenidos realizada en casa por el estudiantado para luego dar paso a la resolución de problemas y cuestiones de forma grupal por parte de los estudiantes. De esta manera, el docente podía moverse libremente por el aula y prestar ayuda a aquellos estudiantes que lo necesitaran. La sesión finalizaba con la puesta en común de la solución por parte del estudiantado y la resolución de dudas por parte del docente. En la última sesión presencial, los estudiantes presentaron los trabajos realizados en grupo y se realizó una encuesta de evaluación acerca de la asignatura. Con dicha encuesta se midió el grado de satisfacción del estudiantado respecto a la asignatura y a la metodología docente empleada utilizando una escala de Likert de 5 niveles y una pregunta para la recogida de comentarios o sugerencias de mejora. Los apartados de la encuesta hacían referencia a la cantidad y calidad de las interacciones estudiante-docente y estudiante-estudiante en el aula, la cantidad y calidad de los materiales disponibles para el estudio, la satisfacción con los resultados 
de aprendizaje obtenidos y la comparación entre la nueva metodología propuesta y la metodología tradicional basada en clases magistrales. Además de dichas encuestas, se estudiaron los resultados de la Encuesta General de Enseñanza realizada por la Unidad Técnica de Calidad de la Universidad de Oviedo.

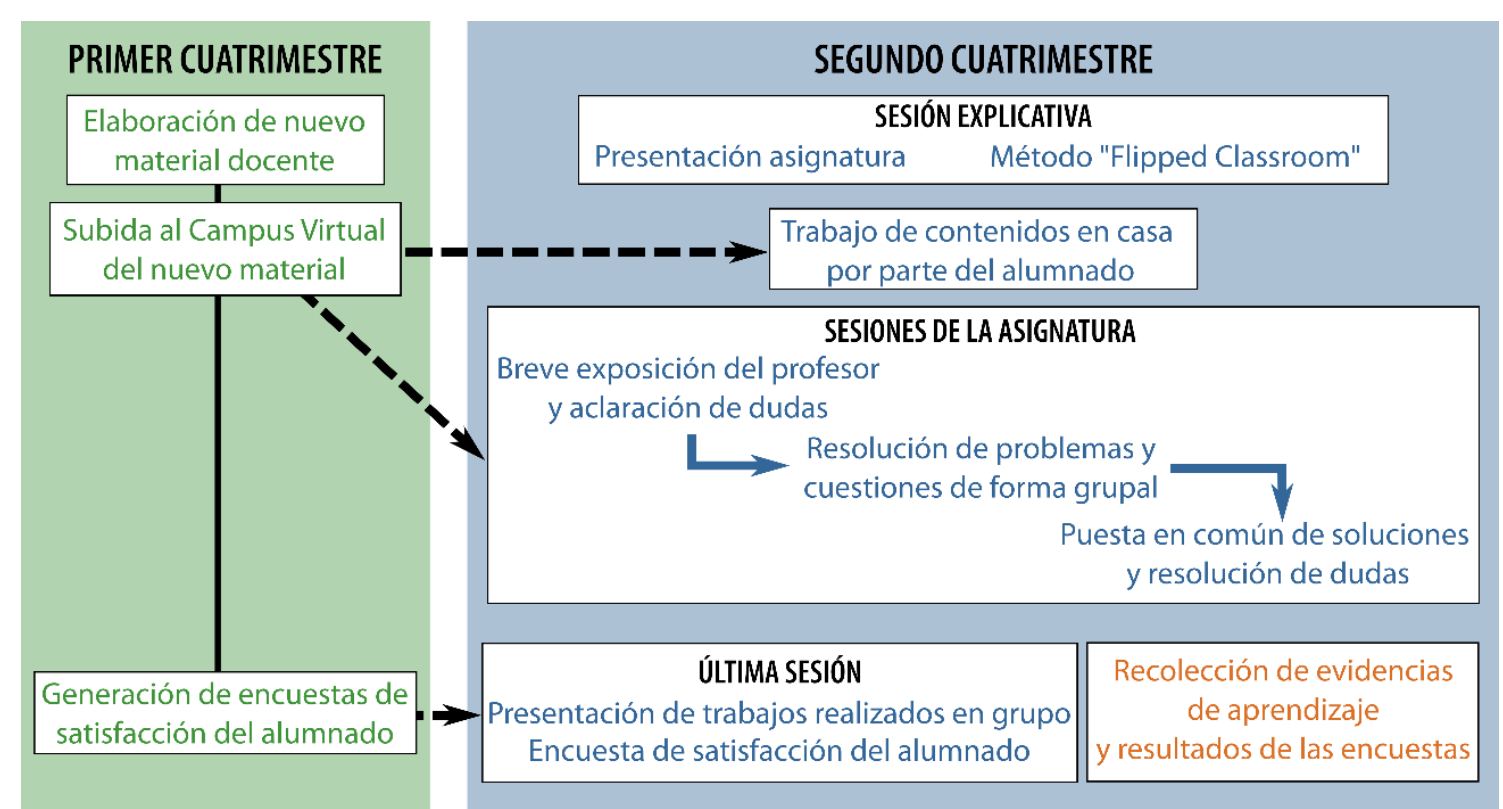

Figura 1. Esquema del desarrollo e implantación en el aula de la experiencia de innovación propuesta (elaboración propia).

\section{RESULTADOS}

En esta sección se analizan los resultados obtenidos con la implantación de la experiencia de innovación desarrollada.

\subsection{Mejora de los resultados de aprendizaje}

La media de las calificaciones obtenidas por el estudiantado en la asignatura pasó de 7,5 a 9,2 , lo que se traduce en una mejora del $23 \%$. Cabe señalar que la desviación típica de las notas se redujo considerablemente, probablemente como consecuencia de las situaciones de aprendizaje propiciadas en el aula en las que se fomentaba la cooperación colaborativa entre el estudiantado. Estos resultados pueden relacionarse directamente con el mejor aprovechamiento del tiempo en el aula como consecuencia de la aplicación de la metodología "flipped clasroom", que permitía al docente atender a las necesidades particulares de aprendizaje de cada estudiante, y el diseño de actividades de aula basadas en la colaboración entre el estudiantado. El número de trabajos aprobados por cada estudiante aumentó de manera considerable, así como la nota de los mismos.

En este sentido, se puede afirmar que la innovación propuesta ha conseguido su objetivo, al conseguir aprovechar mejor el potencial de aprendizaje del estudiantado gracias a una mejor gestión de los tiempos en el aula. 
Tabla 1.

Calificaciones obtenidas por el estudiantado durante los años académicos 2017/18 a 2019/20.

\begin{tabular}{lccc}
\hline \multicolumn{1}{c}{ Curso académico } & $\mathbf{2 0 1 7 / 1 8}$ & $\mathbf{2 0 1 8 / 1 8}$ & $\mathbf{2 0 1 9 / 2 0}$ \\
\hline Nota media & 7,5 & 8,1 & 9,2 \\
\hline Desviación típica & 1,14 & 1,03 & 0,33 \\
\hline
\end{tabular}

Fuente: Elaboración propia

\subsection{Resultados de la Encuesta General de Enseñanza}

Los resultados principales de la Encuesta General de Enseñanza de la Universidad de Oviedo se muestran en la Figura 2. Los datos correspondientes al periodo anterior a la implantación de la innovación propuesta se representan en azul y los datos posteriores a la misma en verde. Los datos correspondientes a la media de la titulación se encuentran representados en naranja. Se puede observar que la satisfacción del estudiantado con la asignatura ha aumentado de manera considerable, así como su percepción acerca del programa formativo, la orientación recibida y las prácticas realizadas. De esta forma, los indicadores de la asignatura han pasado de situarse por debajo de la media a que ésta sea una de las asignaturas mejor valoradas dentro de la titulación.

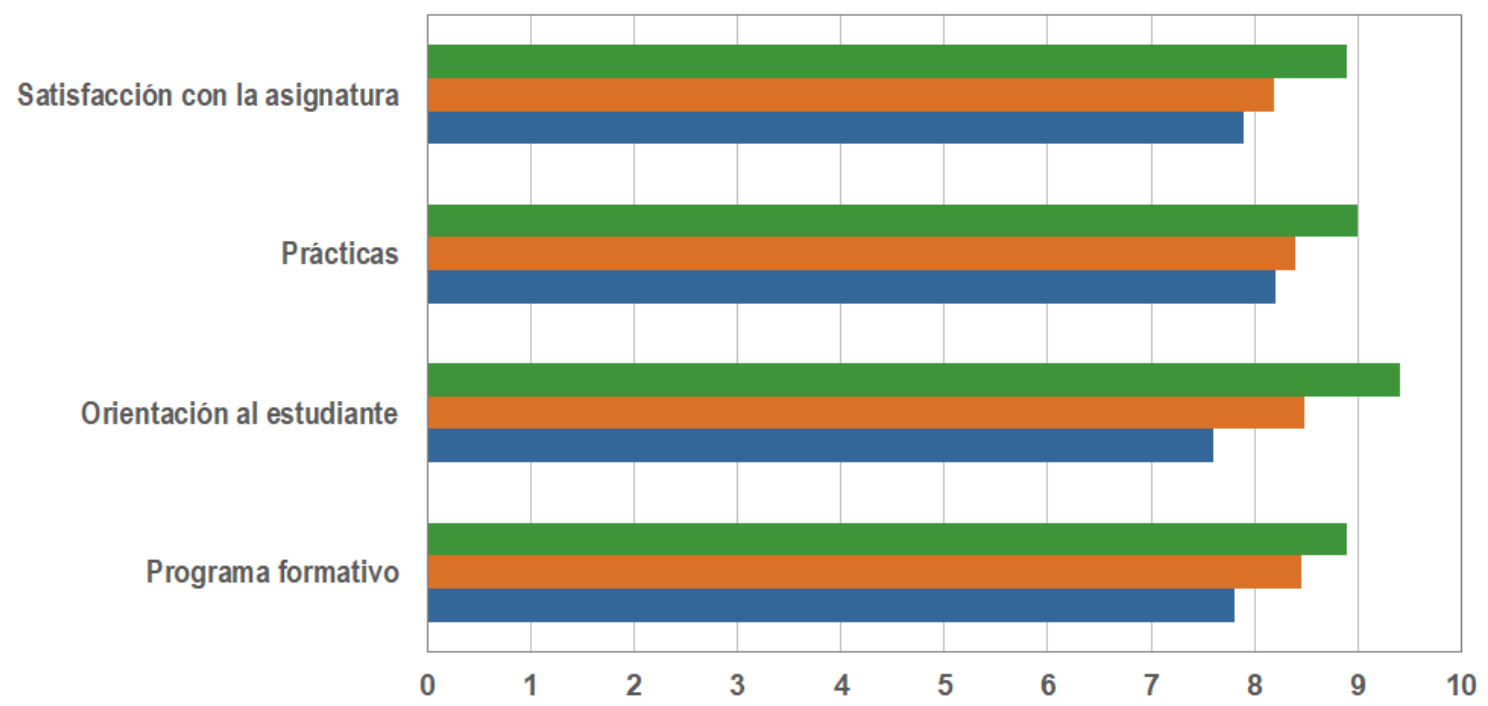

Figura 2. Resultados de la Encuesta General de Enseñanza de la Universidad de Oviedo antes (azul) y después (verde) de la experiencia de innovación desarrollada junto a la media (naranja) de las asignaturas de la titulación (elaboración propia).

\subsection{Resultados de las encuestas sobre la metodología docente empleada}

Los resultados de las encuestas realizadas por el estudiantado fueron muy positivos. Todas las respuestas obtenidas fueron superiores a 4 puntos sobre 5 en la escala propuesta. La Figura 3 muestra los resultados de dichas encuestas, de los que se puede deducir que el estudiantado evaluó de forma positiva la innovación implantada en el aula. La interacción entre iguales (estudiante-estudiante) fue una de las mayores beneficiadas, así como la satisfacción del estudiantado con sus propios resultados de aprendizaje.

En general y como se recogió en varios de los comentarios de las encuestas, el estudiantado tuvo la percepción de que la metodología propuesta resultó muy eficaz. A 
pesar de sentir una mayor obligación de trabajar, sentían mayor confianza en la comprensión que alcanzaban de los contenidos de la asignatura. El poder trabajar de manera colaborativa, además, les permitió hacerse protagonistas de su propio proceso de aprendizaje, mientras el docente tenía un papel de guía y ayuda durante dicho proceso. Finalmente, el estudiantado consideró la asignatura como muy interesante, llegando incluso a comentar en la última sesión que les gustaría que el tiempo de docencia se prolongara más en el tiempo

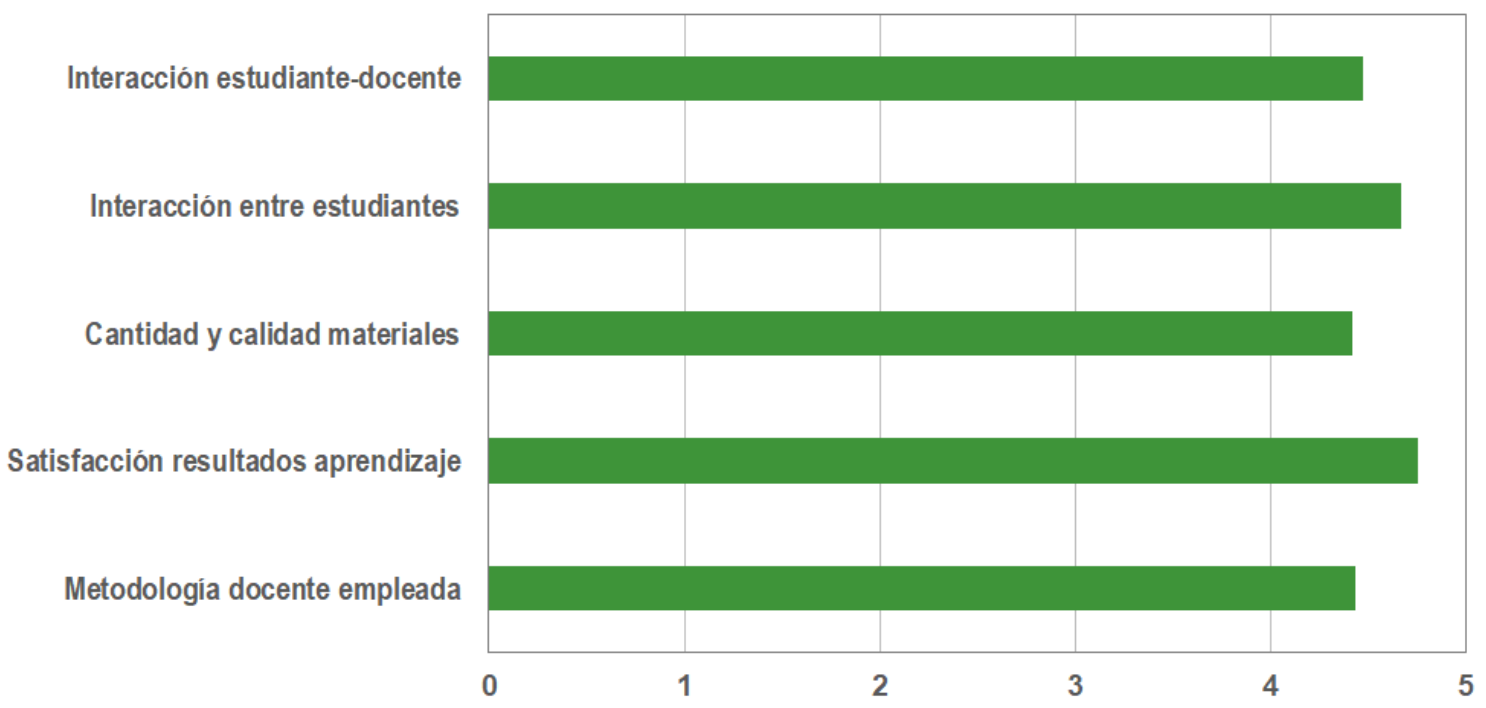

Figura 3. Resultados de la encuesta realizada al estudiantado al final de la experiencia de innovación.

\subsection{Autoevaluación de la mejora implementada}

En este último apartado se recoge la evaluación de la propuesta realizada por los propios investigadores y motivadores de la misma.

Entre las principales fortalezas de la misma, cabe destacar su carácter innovador, así como la coherencia entre sus objetivos y la metodología que se ha empleado. Además, permite que el estudiantado interaccione con el contenido de la asignatura de forma autónoma, facilitando que construya su propio aprendizaje. La propuesta fomenta la colaboración entre los estudiantes y además no se trata de una intervención que requiera una gran cantidad de recursos o lleve asociada un gran coste. Finalmente, el profesorado cuenta con formación adecuada y suficiente para poder llevar la intervención a cabo

En cuanto a las debilidades detectadas, existe una dependencia de la disponibilidad de recursos tecnológicos (principalmente el Campus Virtual). Por otra parte, existe una elevada necesidad de colaboración por parte del estudiantado en el proceso de aprendizaje, dado que sin el trabajo previo en casa resultaría muy difícil llevar el ritmo de las clases. Además, la impartición de la asignatura se realiza de forma muy concentrada (dos semanas), lo que dificulta la aplicación del método

Las principales amenazas a las que se enfrenta la propuesta son las posibles limitaciones de los dispositivos que el estudiantado tenga a disposición en el hogar, así como un posible aumento del ruido y el desorden en el aula. Sin embargo, las oportunidades que se presentan con esta propuesta son bastante más interesantes: la Universidad de Oviedo está totalmente abierta a la innovación y experimentación educativa, fomentándola a través de un programa anual de innovación. Además, los primeros resultados obtenidos muestran que la mejora implementada ha conseguido un aumento tanto en los resultados de aprendizaje como en la satisfacción del estudiantado con la metodología y la asignatura. La metodología propuesta fomenta la reflexión del estudiantado acerca de los contenidos de la asignatura. Cabe destacar, por otra parte, 
que el curso 2020/21 la asignatura se impartirá de manera distribuida a lo largo de todo el semestre, salvando una de las debilidades de la propuesta y de modo que se prevé que la metodología propuesta será más efectiva. Finalmente, resultaría relativamente sencillo extender esta innovación a otras asignaturas del área de conocimiento al que pertenece el equipo investigador

\section{CONCLUSIONES}

Tras observar que parte del potencial del estudiantado se encontraba desaprovechado a la luz de los resultados de aprendizaje observados, se detectó la necesidad de conseguir un mejor aprovechamiento de las clases presenciales a través de la mejora de la gestión del tiempo de aprendizaje. Con este objetivo, se desarrolló un Proyecto de Innovación Docente basado en una metodología "flipped classroom" y de aprendizaje colaborativo. Para ello, se generaron nuevos contenidos teóricos y prácticos (cuestiones y problemas) que se pusieron a disposición del estudiantado a través del Campus Virtual antes de las sesiones de aula. Previamente a las sesiones en el aula, se pedía a los estudiantes que trabajaran una serie de contenidos en casa, y las sesiones de aula consistieron en breves exposiciones por parte del profesor y aclaración de dudas para dar paso a la resolución de problemas y cuestiones de forma colaborativa por parte de los estudiantes. En la última sesión, los estudiantes presentaron los trabajos realizados y realizaron las encuestas de evaluación acerca de la experiencia de innovación implantada.

Los resultados de la puesta en marcha de esta innovación son prometedores, en cuanto se ha conseguido una mejora sustancial de los resultados de aprendizaje obtenidos por el estudiantado (la nota media de la asignatura mejoró en un $23 \%$ respecto al periodo de referencia). Además, los estudiantes revelaron un mayor grado de satisfacción tanto respecto a la asignatura en sí misma y los resultados de aprendizaje alcanzados como respecto al cambio en la metodología docente empleada. Se ha observado que la metodología colaborativa propuesta, basada en el modelo "flipped classroom" no solo contribuye a mejorar de forma cuantitativa los resultados de aprendizaje, sino que permite que los estudiantes se hagan protagonistas de su propio proceso de aprendizaje, consigan más confianza en sí mismos y en su conocimiento y también que mejoren la interacción entre sí mismos y con el docente en el contexto del aula.

Finalmente, a partir de la autoevaluación de la mejora implementada para determinar sus fortalezas y limitaciones, así como posibles propuestas de mejora de cara al futuro, se ha decidido continuar en el siguiente curso académico con la mejora implantada, para poder evaluar su consolidación.

\section{REFERENCIAS}

Acaso, M., Manzanera, P. y Piscitelli, A. (2015). Esto no es una clase. Investigando la educación disruptiva en los contextos educativos formales. Madrid: Fundación Telefónica.

Barrel, J. (2006). Problem-Based Learning: An Inquiry Approach. Reino Unido: Corwin.

Bergmann, J., Sams, A. (2012). Flip your classroom: reach every student in every class every day. International Society for Technology in Education, Association for Supervision and Curriculum Development.

Cohen, E.G., Lotan, R.A. (2014). Designing groupwork - Strategies for the heterogeneous classroom. New York: Teachers College Press. 
Fernández Díaz, M.J. (2005). La innovación como factor de calidad en las organizaciones educativas. Educación XX1, 8, 67-86.

Goodwin, B. y Miller, K. (2013). Research says evidence on Flipped Classrooms is still coming in. Technology Rich Learning, 70(6), 78-80

Iborra Urios, M., Ramírez Range, E., Badia Córcoles, J.H., Bringué Tomàs, R., Tejero Salvador, J. (2017). Implementing the flipped classroom methodology to the subject "Applied Computing" of two engineering degrees at the University of Barcelona. Journal of Technology and Science Education, 7(2), 119-135

Maroto Molina et al. (2017). El método del caso como herramienta para la docencia y el aprendizaje en ingeniería de sistemas de producción ganadera. Revista de Innovación y Buenas Prácticas Docentes, 1, 45-50.

Pierce, R., Fox, J. (2012). Vodcasts and Active-Learning Exercises in a "Flipped Classroom" Model of a Renal Pharmacotherapy Module. American Journal of Pharmaceutical Education, 76(10), Article 196.

Real Decreto 1393/2007, de 29 de octubre, por el que se establece la ordenación de las enseñanzas universitarias oficiales. Boletín Oficial del Estado. Madrid, 30 de octubre de 2007, núm.260, pp. 1-28 (texto consolidado).

Real Decreto 99/2011, de 28 de enero, por el que se regulan las enseñanzas oficiales de doctorado. Boletín Oficial del Estado. Madrid, 10 de febrero de 2011, núm.35, pp. 1-17 (texto consolidado).

Real Decreto 1027/2011, de 15 de julio, por el que se establece el Marco Español de Cualificaciones para la Educación Superior. Boletín Oficial del Estado. Madrid, 3 de agosto de 2011, núm.185, pp. 1-7 (texto consolidado).

Romero, M.C. y Prat, A. (2018). Implementación del modelo Flipped Classroom para la enseñanza de Matemáticas en Educación Secundaria Obligatoria. En I. Cabero y B. Ortega (eds.), Innovaciones Educativas motivadoras del conocimiento de las matemáticas y las ciencias. España: Ediciones Egregius.

Tourón, J. y Santiago, R. (2015). El modelo flipped learning y el desarrollo del talento en la escuela. Revista de Educación, 368, 196-208

Vanwalleghem, T. et al. (2018). Aprendizaje liderado por los estudiantes mediante elaboración de vídeos colaborativos sobre hidrología y erosión. Revista de Innovación y Buenas Prácticas Docentes, 6, 1-5 